\title{
Study on Amino Acids in Ginseng Yanping Zhu Jilin Agricultural University
}

\author{
Yanping Zhu \\ Jilin Agricultural University, Changchun 130118, China
}

Keywords: ginseng; amino acid; composition; analysis.

\begin{abstract}
Ginseng is a rare medicinal medicine, has a long history of medicine. To the modern research on ginseng deepening, especially on the composition of ginseng in-depth analysis. Related research found that ginseng contains a variety of chemical composition, including protein, amino acids, vitamins, alkaloids, ginsenosides, polysaccharides, organic acids and so on. The importance of amino acids for the human body is obvious, because the amino acid is to build cells, the necessary material to grow tissue, but also directly involved in the body's own mediation process, to strengthen the human immune system, to resist disease invasion has a key role. In this paper, the amino acid composition of ginseng was analyzed and studied.
\end{abstract}

\section{Introduction}

Medicinal ginseng generally refers to the root of the five plants plant ginseng root, through the corresponding treatment of the root to remove the water, get dry rhizome, generally do not need to be treated by other treatments can be used as a medicinal material. Ginseng's medicinal history is long, and very early by the medical scientists to recognize its efficient tonic value, it has always been used as a valuable Chinese herbal medicine. It has been found that ginseng contains a variety of chemical constituents, including protein, amino acid, vitamins, alkaloids, ginsenosides, polysaccharides, organic acids and so on. Although the main component of ginseng is protein, but through the modern medical research found that ginseng in the amino acid for the benefit of ginseng play a key role.

At present, there are many analytical methods for amino acid composition in ginseng, and more commonly used include amino acid automatic analysis technology, pre-column derivatization reversed-phase high performance liquid chromatography, high performance liquid chromatographyevaporative light scattering detection, liquid chromatography combined technology The The researchers used amino acid automatic analyzer to analyze the wild ginseng in Changbai Mountain. At the same time, the results were compared with the amino acid components of American ginseng and American ginseng. The results showed that all ginseng contained 17 kinds of basic amino acids, among which seven kinds of amino acids were Human growth and development of indispensable substances, leucine content of the least. The results showed that the amino acid content of ginseng in Changbai Mountain area was higher than that in garden ginseng and American ginseng, and the content of amino acid in wild ginseng increased with the growth period. The use of amino acid automatic analyzer on the analysis of amino acids in ginseng, with more advantages, one of its high degree of automation, the second is a short time required, the third is the analysis of the results with high sensitivity and accuracy, in practical applications But this analysis method also has certain defects and shortcomings, mainly for the analysis process is easy to be affected by the buffer $\mathrm{pH}$ value, in addition to the relatively expensive analytical instruments, analysis will be completed after the discharge of pollution The role of organic solvents. Wang Yuhong et al. Use the Evaporative Light Scattering Detector liquid chromatography-evaporative light scattering detection. This method requires the temperature of the detection environment to be controlled at 40 degrees to analyze the essential amino acids that are not derived from ginseng. Based on the further amino acid injection of the separation and detection, and ultimately get the ginseng amino acid content.High performance liquid chromatography-evaporative light scattering detection method has the advantages of simple operation and accurate detection result in the analysis of amino acid content and composition process, and it does not need to be derivatized to the ginseng before testing, so it can save the detection time effectively and has certain Promote the application value. 


\section{Materials and instruments}

\section{1 herbs}

This study used ginseng to self-Changbai Mountain area and North Korea's ginseng area, by the same department to follow-up treatment to get dry reference.

\subsection{Instruments}

Hitachi 835-50 amino acid automatic analyzer, balance, universal grinder, pure water system, blast oven.

\subsection{Reagents}

Acetonitrile, methanol, ultrapure water, heptafluorobutyric acid, trifloxacin, in addition to 24 amino acid reference products, namely alanine, arginine, aspartic acid, cysteine, cystine, Glutamic acid, histidine, lysine, amino acid, phenylalanine, serine, tryptophan, threonine, tyrosine and the like.

\subsection{Experimental methods}

The ginseng was placed in a pulverizer for crushing, and finally the ginseng powder was obtained through a 60 mesh sieve. $200 \mathrm{mg}$ of ginseng powder was weighed using a balance, and the mixture was placed in a hydrolysis tube while $6 \mathrm{ml}$ of HCI was added inward. The hydrolysis tube into the dry ice, to promote the tube ginseng powder freeze, turn on the vacuum pump, its pumping decompression, when the corresponding standards to achieve the implementation of sealing. The hydrolyzate tube was hydrolyzed at $110^{\circ} \mathrm{C}$ for 24 hours. After completion of hydrolysis, remove the hydrolysis tube, open the seal, the hydrolysis of the filter operation, remove the sample. Put the ginseng powder into the pan and place the dryer together to keep the sample dry. After drying, add 1 $\mathrm{mL}$ of $0.01 \mathrm{~N} \mathrm{NaOH}$ and place the sample in the atmosphere to maintain this state for 4 hours. This process can cause conversion of amino acids in ginseng and conversion from cysteine to cystine. Add one milliliter of $0.1 \mathrm{NHC} 1$ to the sample and add three milliliters of buffer. The buffer can dilute the sample, helping to complete the test and improve the detection accuracy. The residue in the sample was treated with a glass rod, and further filtration was carried out using a filter paper, and the buffer was washed again with the buffer. The concentration of the test solution is controlled at $20 \mathrm{mg}$ per milliliter, and the sample is prepared and sealed, and then stored in a refrigerator for later use. The samples were analyzed by Hitachi 835-50 amino acid automatic analyzer. The analytical factors are as follows: Alkaline column: column diameter $9 \mathrm{~mm}$, resin height $70 \pm 5 \mathrm{~mm}$, analysis time $80 \pm 10$ minutes, buffer $\mathrm{pH} 5.25$, buffer flow rate $60 \mathrm{ml} / \mathrm{h}$, buffer pump pressure $3-8 \mathrm{Kg} / \mathrm{Cm} 2$, column temperature of 55 degrees, reaction temperature of 100 degrees, injection volume of $0.01-0.4 \mathrm{uM} /$ $0.5 \mathrm{ml}$, nitrogen pressure of $1 \mathrm{Kg} / \mathrm{cm} 2$, paper speed of $180 \mathrm{~mm} / \mathrm{h}$. Acid neutral column: Column diameter $9 \mathrm{~mm}$, resin height $540 \pm 10 \mathrm{~mm}$, analysis time $190 \pm 20$ minutes, buffer $\mathrm{pH} 3.25,4.25$, buffer flow rate $60 \mathrm{ml} / \mathrm{h}$, buffer pump pressure $8-15 \mathrm{Kg} / \mathrm{cm} 2$, The column temperature is 55 degrees, the reaction temperature is 100 degrees, the injection volume is $0.01-0.4 \mathrm{uM} / 0.5 \mathrm{ml}$, the buffer replacement time is 93 minutes, the nitrogen pressure is $1 \mathrm{Kg} / \mathrm{cm} 2$ and the paper speed is $180 \mathrm{~mm} /$ h.

According to the existing mixed amino acid standard map, with reference to the amino acid known concentration standard peak area and the unknown concentration of the peak area ratio, the final accurate calculation of the sample of various amino acids in the composition and content. In the calculation process, the sample weight and water content need to be taken into account, so as to effectively ensure the accuracy of the percentage of amino acids.

\section{Analysis of amino acid composition in ginseng}

In this study, three kinds of ginseng were selected, which were the six-year rotation of the old Senate, the soil of the new forest land and the soil reference. The following results were obtained by analyzing the amino acids automatically.

Agaric acid 0, lysine 0.84, histidine 0.46, arginine 3.85, basic amino acid content of 5.99, aspartic acid 1.05, threonine 0.39, serine 0.31, valley Amino acids 1.73 , proline 0.85 , glycine 0.38 , alanine 0.51 , cystine 0 , valine 0.42 , methionine 0 , isoleucine 0.36 , leucine 0.92 , tyrosine 0.14 , phenylalanine 
0.48 , the total amount of acid neutral amino acids was 7.54 , and the total amount of amino acids was 13.53

Lysine 0, lysine 0.75 , histidine 0.37 , arginine 3.25 , basic amino acid content 5.35 , aspartic acid 0.94 , threonine 0.30 , serine 0.27 , glutamic acid 1.92 , Proline 0.38 , glycine 0.31 , alanine 0.40 , cystine 0 , valine 0.39 , methionine 0 , isoleucine 0.35 , leucine 0.64 , tyrosine 0.14 , phenylalanine 0.44 , acid The total amount of neutral amino acid was 6.48 and the total amino acid was 11.83.

The contents of tryptophan 0 , lysine 0.67 , histidine 0.39 , arginine 4.19 , basic amino acid content 6.02, aspartic acid 0.95, threonine 0.36 , serine 0.27 , glutamic acid 1.732 , Proline 0.35 , glycine 0.32 , alanine 0.42 , cystine 0 , valine 0.40 , methionine 0 , isoleucine 0.32 , leucine 0.60 , tyrosine 0.16 , phenylalanine 0.42 , The total amount of amino acids was 5.89 , and the total amount of amino acids was 11.91.

\section{Discussion}

Ginseng chemical composition is very complex, the study found that the main component of ginseng protein, in addition also contains ginsenosides, sugars, amino acids, vitamins and other nutrients.

Ginseng for the human body has a strong tonic effect, can effectively regulate the body's blood pressure, strengthen the heart function, but also can improve the symptoms of neurasthenia, can play the role of qi body strength. Modern pharmacological studies have found that ginseng substances in the central nervous system can play a role in the excitement and inhibition, which makes ginseng can effectively enhance the human nerve activity excitement and inhibition process, while effectively strengthening the body's external environment adaptability, and ultimately enhance the body The function, reduce fatigue. In addition, ginseng can also be on the human heart, myocardium, blood vessels and other vital organs play a protective role.

The important role of ginseng is closely related to its chemical composition. Ginseng contains many kinds of chemical substances. In this paper, the amino acid composition of ginseng is studied deeply. The results show that the ginseng produced in three different soil areas is rich in amino acids, And the like, including tryptophan, lysine, histidine, arginine, aspartic acid, threonine, serine, glutamic acid, proline, glycine, alanine, , Valine, isoleucine, leucine, tyrosine, phenylalanine and the like.

The results showed that the total amount of ginseng amino acid was $13.53 \%$ for the six years, while the content of amino acids in the soil and soil of the new forest land was $11.83 \%$ and $11.9 \%$, respectively. The results showed that the amino acid content of ginseng was more than that of the other two kinds of ginseng, but the three kinds of ginseng had no obvious difference in amino acid composition and species. The total amount of neutral amino acid was 7.54, the total amount of amino acid was 13.53, the content of amino acid in the new forest land was 5.35, the total amount of acid neutral amino acid was 6.48 , the total amount of amino acid was 11.83 , the content of alkaline earth amino acid was 6.02 , The total amount of acid neutral amino acids was 5.89, and the total amount of amino acids was 11.91. The above results showed that the content of reference amino acid was higher than that of the other two kinds of ginseng. Further analysis of the composition of amino acids in ginseng found that ginseng also contains the human body can not successfully synthesize their amino acids such as lysine, valine, leucine, acid and phenylalanine, which is essential for the human body Of the nutrients, it can be found that ginseng on the human body a lot of tonic from the role of these amino acids.

\section{References}

[1]. Yang Kaihua, Bian Qingquan, Lu Rui, et al. Determination of Amino Acids in Gin seng Fruit [J]. Food Research and Development, 2015 (11): 120-122. 
[2]. Lv Jingnan, Luo Haoming, Chen Yinghong, et al. Screening and structural characterization of active ingredients in the treatment of senile dementia with ginsenoside saponin [J] .Journal of Chinese Medicine, 2015 (11): 2561-2563.

[3]. Zhang Aihua, An Ningbo, Lei Fengjie, etc. Bacterial soft rot fungi on ginseng root exudates of sugar and amino acids in the chemical trend response [J]. Chinese Journal of Traditional Chinese Medicine, 2016, 41 (21): 3937 -3941.

[4]. YANG Yan-wen, JIANG Yuan-tong, ZHANG Lian-xue.Comparison of Soil Enzyme Index and Chemical Components in Rhizosphere Soil of Farmland and Senate [J] .Hubei Horticulture, 2016 (17): 157-162.

[5]. Song Juan, Lei Xiujuan, Yin Hongxin, et al.Comparison of Amino Acids in Callus of F_1 Generation and Parents of Panax ginseng [J]. Northern Gardening, 2016 (16): 149-151.

[6]. Cao Limin, Long Chunlin, Cao Ming. Analysis of Nutritional Components of Two Kinds of Healthy Vegetables in Gannan, Jiangxi Province [J] .Chinese Journal of Medicine, 2015 (11): 2597-2599. 